\title{
基于甲胺客体调制的混价态甲酸铁框架的构筑与磁电行为研究
}

\author{
赵畑鹏 ${ }^{a, c}$ 王玮玮 ${ }^{a}$ 韩松德 $^{b}$ 李泉文 ${ }^{c}$ 李娜 ${ }^{c}$ \\ 刘福臣*,a显和 $*, b, c$ \\ ( ${ }^{a}$ 天津理工大学 化学化工学院 天津 300384) \\ ( ${ }^{b}$ 南开大学 化学学院 元素有机化学国家重点实验室 天津 300071) \\ ( ${ }^{c}$ 南开大学 材料科学与工程学院 天津市金属与分子基材料化学重点实验室 天津 300350)
}

\begin{abstract}
摘要 $N$-型亚铁磁体可以在场冷时呈现负磁化行为, 但在 $1 \mathrm{~T}$ 强场场冷时依然能够保持负磁化行为的化合物却很罕见. 本工作以 $\mathrm{CH}_{3} \mathrm{NH}_{3}{ }^{+}$为客体阳离子, 成功构筑了一例红砷镍矿 $\left(4^{9} .6^{6}\right)\left(4^{12} .6^{3}\right)$ 结构的混价态甲酸铁化合物 $\left[\mathrm{CH}_{3} \mathrm{NH}_{3}\right]_{n}\left[\mathrm{Fe}^{\mathrm{III}} \mathrm{Fe}^{\mathrm{III}}\left(\mathrm{HCO}_{2}\right)_{6}\right]_{n}(\mathbf{1})$, 在 $1 \mathrm{~T}$ 强场下呈现负磁化行为. 1 中甲酸根采用反, 反模式连接 $\mathrm{Fe}^{\mathrm{II}}$ 和 $\mathrm{Fe} \mathrm{el}^{\mathrm{III}}$ 形成三维阴离 子框架, 八面体配位构型的 $\mathrm{Fe}^{\mathrm{II}}$ 和 $\mathrm{Fe} \mathrm{III}^{\mathrm{II}}$ 分别处于 $\left(4^{9} \cdot 6^{6}\right)$ 和 $\left(4^{12} .6^{3}\right)$ 节点, 而 $\mathrm{CH}_{3} \mathrm{NH}_{3}{ }^{+}$则填充在框架空隙中平衡电荷. 量热、 介电和单晶 X-射线衍射测试结果表明, $\mathbf{1}$ 中存在由 $\mathrm{CH}_{3} \mathrm{NH}_{3}{ }^{+}$无序-有序转变诱导的结构相变并伴随介电驰豫. 磁性研究 表明 $\mathrm{Fe}^{\mathrm{II}}$ 和 $\mathrm{Fe}^{\mathrm{III}}$ 之间存在较强的反铁磁耦合, 但在冷却过程中 $\mathrm{Fe}^{\mathrm{II}}$ 和 $\mathrm{Fe} \mathrm{III}^{\mathrm{II}}$ 构成的亚晶格磁有序程度存在较大差异, 且具 有较小自旋的 $\mathrm{Fe}^{\mathrm{II}}$ 磁矩先于 $\mathrm{Fe}^{\mathrm{III}}$ 磁矩有序且平行于外加磁场, 导致温度进一步降低时 $\mathbf{1}$ 呈现出负磁化行为, 这表明 $\mathbf{1}$ 是一例罕见的 $N$-型亚铁磁体. 此外, 1 在 $1 \mathrm{~T}$ 外加冷却场中依然能保持负磁化行为, 表明 1 中存在较强的磁各向异性. 值 得注意的是, 在 $100 \mathrm{Oe}$ 外场下冷却到 $17 \mathrm{~K}$ 后, 1 呈现出正场调制的磁极翻转行为. 进一步研究表明, $\mathbf{1}$ 的场冷磁滞回线 呈现不对称形状, 并向冷却场方向发生偏转, 其有效矫顽力 $\left(H_{\mathrm{C}}{ }^{\mathrm{eff}}=\left(H^{+}-H^{-}\right) / 2\right)$ 和交换偏置场 $\left(H_{\mathrm{EB}}=\left(H^{+}+H^{-}\right) / 2\right)$ 分别 为 $21716 \mathrm{Oe}$ 和 $3322 \mathrm{Oe}$. 总之, 本研究表明客体分子尺寸和形状能够有效调控红砷镍矿结构中 $\mathrm{Fe}^{\mathrm{II}}$ 的磁各向异性, 从而 实现较高的磁极翻转场、矫顽力和交换偏置场.
\end{abstract}

关键词 混价态; 相变; 负磁化; 磁极翻转; 交换偏置

\section{Construction, Magnetic and Dielectric Properties of Mixed-Valence Iron Formate with Methylammonium Guest}
Zhao, Jiongpeng ${ }^{a, c}$
Wang, Weiwei ${ }^{a}$
Liu, Fuchen*,a
Han, Songde ${ }^{b}$ $\mathrm{Bu}, \mathrm{Xianhe}^{*, b, c}$
Li, Quanwen ${ }^{c}$
$\mathrm{Li}, \mathrm{Na}^{\mathrm{c}}$ \\ ( ${ }^{a}$ School of Chemistry and Chemical Engineering, Tianjin University of Technology, Tianjin 300384, China) \\ ( ${ }^{b}$ State Key Laboratory of Elemento-Organic Chemistry, College of Chemistry, Nankai University, Tianjin 300071, China) \\ ( ${ }^{c}$ Tianjin Key Laboratory of Metal and Molecule-Based Material Chemistry, School of Materials Science and \\ Engineering, Nankai University, Tianjin 300350, China)
}

Abstract Although $N$-type ferrimagnets exhibit negative magnetization under positive magnetic fields, compounds could maintain negative magnetization behavior under strong magnetic field (e.g. $1 \mathrm{~T})$ are still rare. In this work, a mixed-valence metal-formate $\left[\mathrm{CH}_{3} \mathrm{NH}_{3}\right]_{n}\left[\mathrm{Fe}^{\mathrm{III}} \mathrm{Fe}^{\mathrm{II}}\left(\mathrm{HCO}_{2}\right)_{6}\right]_{n}(\mathbf{1})$ was constructed by the reaction of $\mathrm{FeCl}_{3} \cdot 6 \mathrm{H}_{2} \mathrm{O}$, formic acid and $N$-methyl formamide at $140{ }^{\circ} \mathrm{C}$ for two days. At room temperature, 1 crystallizes in the space group $P \overline{3} 1 \mathrm{c}$, in which a three-dimensional anionic niccolite topological framework is constructed by $\mathrm{Fe}^{\mathrm{II}}, \mathrm{Fe}^{\mathrm{III}}$ ions, and anti,anti formate. The guest $\mathrm{CH}_{3} \mathrm{NH}_{3}{ }^{+}$cations fill in the cavities of the framework as the charge balancer with the $\mathrm{N}$ atoms displaying threefold disorder and the atoms having a twofold disorder. The heat capacity measurement shows two different peaks, being the signatures of phase transitions. The change in heat capacity at $136 \mathrm{~K}$ corresponds to the phase transition triggered by the order-disorder phenomena of the $\mathrm{CH}_{3} \mathrm{NH}_{3}{ }^{+}$cations. At low temperature phase, $\mathbf{1}$ has a symmetry of $2 / \mathrm{m}$ in space group $C 2 / c$, in which the threefold disorder of the $\mathrm{N}$ atoms of the $\mathrm{CH}_{3} \mathrm{NH}_{3}{ }^{+}$was freezed. The order-disorder phase transition also results in dielectric relaxation in the temperature range $130 \sim 200 \mathrm{~K}$ at $500 \mathrm{~Hz} \sim 1 \mathrm{MHz}$. The change of heat capacity at $40 \mathrm{~K}$ is associated with the ferromagnetic order of the antiferromagnetically coupled $\mathrm{Fe}^{\mathrm{III}}$ and $\mathrm{Fe}^{\mathrm{II}}$ sublattices. $\mathbf{1}$ is a $N$-type ferrimagnet with negative magnetization below $T_{\mathrm{N}}$ on cooling under the applied field, and thermo-driven magnetic poles reversal could be found in 1 with large applied field of $1 \mathrm{~T}$. Furthermore, positive field regulated switchable magnetic dipoles switching of the magnetization, together with obvious huge positive exchange bias is also observed in $\mathbf{1}$. These results reveal the significant magnetic

*E-mail: fcliu@tjut.edu.cn; buxh@nankai.edu.cn

Received August 2, 2020; published September 16, 2020.

Supporting information for this article is available free of charge via the Internet at http://sioc-journal.cn

Project supported by the National Natural Science Foundation of China (Nos. 22035003, 21531005, 21871209, 21571139).

项目受国家自然科学基金(Nos. 22035003, 21531005, 21871209, 21571139)资助. 
anisotropy in 1, and the guest in the framework not only can tune the structural phase transitions but also modulate the anisotropy of the host framework leading to different magnetism.

Keywords mixed-valence; phase transitions; negative magnetization; switchable magnetic dipoles; exchange bias

\section{1 引言}

通常, 顺磁、铁磁、亚铁磁物质在磁场下呈现出正 磁化强度, 其净磁矩平行于磁场排列; 而抗磁性物质和 超导体可以在抵抗外磁场作用下呈现负磁化率. 然而, 有一类特殊的磁有序材料, 其磁化率在场冷却过程中的 有序温度下可以出现由正到负的转变 ${ }^{[1]}$. 这种特殊磁化 行为在磁存储、自旋分辨、磁保温等方面具有潜在应 用 ${ }^{[2]}$. 尽管 Néel 早在 1948 年于亚铁磁唯相理论中就预 测了这种具有负磁化行为的亚铁磁体(并归为 $N$ 型 ${ }^{[3]}$ ), 但截至目前 $N$ 型亚铁磁体依然非常少见, 并且在较大外 场下能够保持负磁化状态的磁性材料更为罕见 ${ }^{[4]}$. 负磁 化的出现与晶体结构、自旋载体的磁矩、磁各向异性、 磁交换以及温度依赖的亚晶格有序等因素密切相关, 内 在机制复杂, 这为相关化合物的构筑与性能调控带来困 难. 因此, 如何构筑在较大磁场 $(>1 \mathrm{~T})$ 下负磁化行为的 磁体依然是一大挑战 ${ }^{[2,5]}$.

近年来, 人们在分子基磁性材料中发现了部分具有 负磁化行为的化合物, 其形成机制各异. 首先, 在以 $\left(\mathrm{Fe}^{\mathrm{II}}{ }_{x} \mathrm{Mn}^{\mathrm{II}}{ }_{1-x}\right)_{1.5}\left[\mathrm{Cr}^{\mathrm{III}}(\mathrm{CN})_{6}\right] \cdot z \mathrm{H}_{2} \mathrm{O}$ 为代表的多金属普鲁士 蓝类化合物 ${ }^{[6]}$ 中, 金属离子之间存在铁磁、反铁磁作用, 不同金属的磁有序差异导致负磁化行为. 并且, 这类普 鲁士蓝类化合物具有极强的化学可调性, 通过不同金属 离子置换即可调控获得多次磁极翻转行为 ${ }^{[7]}$. 然而, 这 类化合物缺乏足够的磁各向异性, 只在较低外加磁场下 表现出负磁化行为.

另一类具有负磁化行为的分子基化合物是层状混 价态草酸铁化合物 $\left(\mathrm{XR}_{4}\right) \mathrm{Fe}^{\mathrm{II}} \mathrm{Fe}^{\mathrm{III}}\left(\mathrm{C}_{2} \mathrm{O}_{4}\right)$. 其中, 部分化合 物可以在 $1 \mathrm{~T}$ 外场下呈现负磁化行为, 但它们缺乏明确 的结构, 其磁行为高度依赖于合成条件 ${ }^{[8]}$. 理论计算表 明, 此类化合物中的负磁化行为和 $\mathrm{Fe} \mathrm{e}^{\mathrm{II}}$ 的轨道角动量在 磁场下的分量密切相关 ${ }^{[9]}$, 即在场冷条件下, $\mathrm{Fe}^{\mathrm{II}}$ 的轨道 角动量产生的磁矩优先和外界磁场相平行, 使得相关化 合物能在外场下保持负磁化行为. 在前期研究中, 我们 进行了混价态/混金属结构砷化镍甲酸盐的研究 ${ }^{[10-14]}$, 在具有负磁化行为红砷镍矿结构的混价态甲酸铁中观 测到交换偏置现象 ${ }^{[10]}$, 并用中子衍射确认冷却过程中 $\mathrm{Fe}^{\mathrm{II}}$ 亚晶格磁矩先于 $\mathrm{Fe}^{\mathrm{III}}$ 有序, 进一步证实了 $\mathrm{Néel}$ 关于 $N$-型亚铁磁体假说 ${ }^{[11]}$; 并在同构化合物中发现多重磁 电双稳态 ${ }^{[12]}$. 这些研究表明负磁化行为极有可能出现 在混价态 $\mathrm{Fe}^{\mathrm{II}} \mathrm{Fe} \mathrm{III}^{\mathrm{III}}$ 化合物中. 通过分子设计策略, 修饰配 位环境, 有望对负磁化等磁性行为进行调控 ${ }^{[13]}$ (图 1).

为获得具有较大翻转场的 $N$-型亚铁磁体, 可以对 砷化镍结构混价态甲酸铁进行结构微调, 改变 $\mathrm{Fe}^{\mathrm{II}}$ 的各 向异性调控其磁性能. 本工作中, 以最小的有机胺阳离
子 $\mathrm{CH}_{3} \mathrm{NH}_{3}{ }^{+}$(MA)为模板构筑了一例具有砷化镍拓扑 结构的化合物 $[\mathrm{MA}]_{n}\left[\mathrm{Fe}^{\mathrm{III}} \mathrm{Fe}^{\mathrm{II}}\left(\mathrm{HCO}_{2}\right)_{6}\right]_{n}$ (1), 并获得了厘 米级大尺寸晶体. X-射线单晶衍射表明室温下化合物结 晶于三方晶系空间群 $P \overline{3} 1 c$, 比热测试表明 $\mathbf{1}$ 在 $136 \mathrm{~K}$ 和 $40 \mathrm{~K}$ 有结构相变和磁相变. 低温单晶结构解析表明 化合物在 $113 \mathrm{~K}$ 具有更低的对称性, 结晶于 $C 2 / c$ 空间 群. 在高低温相中, 甲胺分子中的 $\mathrm{N}$ 原子在三个无序位 置上有明显不同. 变温介电测试揭示 $\mathbf{1}$ 中存在介电弛豫, 进一步证实存在结构相变. 磁性测试表明 1 是一例 $N$ 型亚铁磁体, 磁有序温度 $T_{\mathrm{N}}$ 等于 $40 \mathrm{~K}$, 并在 $1 \mathrm{~T}$ 冷却外 场下依然能保持负磁化行为. 特别是 $\mathbf{1}$ 在 $100 \mathrm{Oe}$ 外场冷 却到 $17 \mathrm{~K}$ 后, 呈现出正场调制的磁极翻转行为. 进一步 研究表明, 1 的场冷磁滞回线具有不对称形状, 并向冷 却场方向发生偏转, 其有效矫顽力 $\left(H_{\mathrm{C}}{ }^{\mathrm{eff}}=\left(H^{+}-H^{-}\right) / 2\right)$ 和交换偏置场 $\left(H_{\mathrm{EB}}=\left(H^{+}+H^{-}\right) /\right) 2$ 分别为 $21716 \mathrm{Oe}$ 和 3322 Oe.



图 1 有机胺调制砷化镍甲酸铁框架中 $\mathrm{Fe}^{\mathrm{II}}$ 的配位环境及其在 $1 \mathrm{~T}$ 强 磁场冷却下的正负磁化状态示意图.

Figure 1 Coordination environment of $\mathrm{Fe}^{\mathrm{II}}$ in an amine-modulated niccolite structural framework and its positive and negative magnetized state under strong field-cooled magnetization (1 T).

\section{2 结果与讨论}

\section{1 相变与介电性质}

将 $0.831 \mathrm{~g}$ 三氯化铁和 $15 \mathrm{~mL}$ 甲酸、 $N$-甲基甲酰胺 的混合溶剂(体积比为 $1: 1$ )装入 $25 \mathrm{~mL}$ 反应釜中, 在 $140{ }^{\circ} \mathrm{C}$ 中加热 $2 \mathrm{~d}$, 便获得化合物 $\mathbf{1}$ 的晶体, 晶体大小可 接近厘米尺寸, 其相纯度由粉末 X-射线衍射确认(图 2a). 比热容测试结果显示 1 在 2 273 K 之间有两个峰 (图 2b), 表明 $\mathbf{1}$ 中存在结构相变和磁相变: 在 $136 \mathrm{~K}$ 的 峰是有机胺有序-无序引起的结构相变, 而 $40 \mathrm{~K}$ 的峰则 代表磁相变. 1 的结构相变温度低于我们以前报道的 $\left[\mathrm{CH}_{3} \mathrm{NH}_{2} \mathrm{CH}_{3}\right]_{n}\left[\mathrm{Fe}^{\mathrm{III}} \mathrm{Fe}^{\mathrm{II}}\left(\mathrm{HCO}_{2}\right)_{6}\right]_{n}\left(\mathrm{DMAFe}^{\mathrm{III}} \mathrm{Fe}^{\mathrm{II}}\right)$ 的 $155 \mathrm{~K}$ (DMA $=\mathrm{CH}_{3} \mathrm{NH}_{2} \mathrm{CH}_{3}{ }^{+}$), 并远低于 $\left[\mathrm{CH}_{3} \mathrm{CH}_{2} \mathrm{NH}_{2} \mathrm{CH}_{2}-\right.$ $\left.\mathrm{CH}_{3}\right]_{n}\left[\mathrm{Fe}^{\mathrm{III}} \mathrm{Fe}^{\mathrm{II}}\left(\mathrm{HCO}_{2}\right)_{6}\right]_{n}\left(\mathrm{DEAFe}^{\mathrm{III}} \mathrm{Fe}^{\mathrm{II}}\right)$ 的 $234 \mathrm{~K}(\mathrm{DEA}=$ 
$\left.\mathrm{CH}_{3} \mathrm{CH}_{2} \mathrm{NH}_{2} \mathrm{CH}_{2} \mathrm{CH}_{3}{ }^{+}\right)^{[10,12]}$, 这主要归因于甲胺阳离子 的质量低于 DMA 和 DEA. 通过介电测试进一步确认了 $136 \mathrm{~K}$ 处存在结构相变. 从图 $2 \mathrm{c}$ 可以看出, 在 $-150{ }^{\circ} \mathrm{C}$ 到一 $60{ }^{\circ} \mathrm{C}$ 之间 $\varepsilon^{\prime}$ 存在一个跃迁台阶, 对应高低介电态 切换. 同时 $\varepsilon^{\prime}$ 台阶具有明显的频率依赖, 其跃迁温度随 频率增加而向高温区移动. 其频率依赖行为从介电损耗 $\tan \delta$ 图(图 2c) 中更能体现出来: 随频率增加, $\tan \delta$ 的峰 值温度由 $500 \mathrm{~Hz}$ 时的 $130 \mathrm{~K}$ 移动到 $1 \mathrm{MHz}$ 时的 $190 \mathrm{~K}$. 介电变化反映出化合物中偶极子的运动. 在介电损耗的 峰值温度处, 偶极子运动速度和频率相同, 此时 $\omega^{-1}=$ $2 \pi v$. 损耗的峰值温度和频率的关系符合 Arrhenius 公式 (图 $2 \mathrm{~d}$ ), 拟合得到 $E_{\mathrm{a}} / k_{\mathrm{B}}=0.28 \mathrm{eV}$, 该数值与文献报道的 其它有机胺为模板的金属甲酸盐数值接近 ${ }^{[15,16]}$.

\section{2 晶体结构}

室温下单晶结构分析表明, 化合物 $\mathbf{1}$ 结晶于三方晶 系 $P \overline{3} 1 c$ 空间群, 其晶胞参数为 $a^{\mathrm{HT}}=b^{\mathrm{HT}}=0.82521(12)$ $\mathrm{nm}, c^{\mathrm{HT}}=1.4164(3) \mathrm{nm}, V^{\mathrm{HT}}=0.8353(2) \mathrm{nm}^{3} .1$ 的不对称 单元中包含一个 $\mathrm{Fe}^{\mathrm{II}}(\mathrm{Fe} 1) 、$ 、个 $\mathrm{Fe}^{\mathrm{III}}(\mathrm{Fe} 2)$ 、一个甲酸根 和一个甲胺阳离子. 甲酸根采用 anti, anti 配位模式连接 $\mathrm{Fe} 1$ 和 Fe2, 从而形成一个砷化镍 $\left(4^{9} .6^{6}\right)\left(4^{12} .6^{3}\right)$ 拓扑结构, 甲胺阳离子处于甲酸和铁离子形成的空隙中(图 3a). Fe1 和 $\mathrm{Fe} 2$ 分别处于 $\left(4^{9} .6^{6}\right)$ 和 $\left(4^{12} .6^{3}\right)$ 拓扑节点, 与 6 个氧原子 配位 $(\mathrm{Fe} 1-\mathrm{O} 1=0.2132(3) \mathrm{nm}, \mathrm{Fe} 2-\mathrm{O} 2=0.2019(3) \mathrm{nm}$ )形 成八面体配位构型(图 $3 b$ ). 二价 $\mathrm{Fe} 1$ 和三价 $\mathrm{Fe} 2$ 的价键

a)

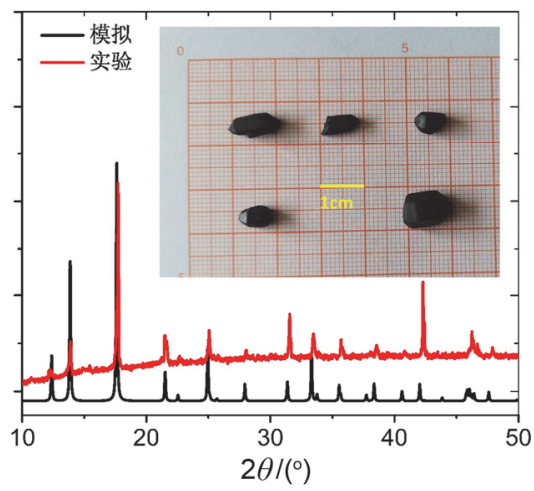

计算结果为 2.05 和 2.98 , 进一步证实了化合物中 $\mathrm{Fe}$ 离 子的价态分布 ${ }^{[17]}$. 每个 $\mathrm{Fe}^{\mathrm{II}}$ 中心周围由甲酸连接六个 $\mathrm{Fe}^{\mathrm{III}}$ 离子形成超级三棱柱结构(图 $3 b$ ), 同时, $c$ 方向上不 同层间相邻的三棱柱通过共边相连接(图 $3 \mathrm{c}$ ), 并以 $\mathrm{ABAB}$ 的堆积方式链接形成三维结构(图 3d). 甲胺阳离 子位于同层三棱柱间隙中, 既是客体也同时起到抗衡离 子作用. 每个甲胺阳离子的 $\mathrm{N}$ 处于一个三重无序状态, 而三个无序的 $\mathrm{N}$ 原子处于 004 面上. 其中, 碳原子处于 一个二重无序状态，位于 004 面的两侧(图 3e, 3f). 比热 容和介电测试结果表明 $\mathbf{1}$ 在 $136 \mathrm{~K}$ 存在结构相变, 因此 在 $113 \mathrm{~K}$ 对 1 进行了单晶 X-射线衍射分析. 结果表明, 在该温度下, 1 的衍射数据可以按照 $C 2 / c$ 空间群进行处 理, 其晶胞参数为: $a^{\mathrm{LT}}=0.8241(6) \mathrm{nm}, b^{\mathrm{LT}}=1.4237(6)$ $\mathrm{nm}, c^{\mathrm{LT}}=1.4001(11) \mathrm{nm}, \beta=90.24(4)^{\circ}, V^{\mathrm{LT}}=1.6427(19)$ $\mathrm{nm}^{3}$ (图 $3 \mathrm{~g}$ ). 据对称性关系可知: $a^{\mathrm{LT}}=a^{\mathrm{HT}}, b^{\mathrm{LT}}=3^{1 / 2} b^{\mathrm{HT}}$, $c^{\mathrm{LT}}=c^{\mathrm{HT}}, V^{\mathrm{LT}}=2 V^{\mathrm{HT}}$. 在低温结构中 $\mathrm{Fe} 1-\mathrm{O}$ 键长在 $0.2113(3) \sim 0.2125(4) \mathrm{nm}$ 之间, $\mathrm{Fe} 2-\mathrm{O}$ 键长在 $0.2004(3) \sim 0.2007(3) \mathrm{nm}$ 之间, 表明结构相变对铁离子 的配位环境影响较小，其价态在结构变化过程中没有发 生明显变化. 但低温时 MA 的位置和室温时相比具有明 显差异, MA 处于 100 面上, 其 $\mathrm{N}$ 原子处于 004 面和 100 面的交汇处，高温的三重无序态变为低温有序态，碳原 子依然具有两重无序位于 004 面的两侧(图 3h, 3i). 从图 中可以看到低温相 1 中作为子晶格偶极子的甲胺在 $b$ 方


图 2 (a) 1 的粉末 X-射线衍射谱图, 插图为 1 的大单晶光学照片. (b) 1 的比热容测试. (c) 1 在不同频率和温度下的介电常数 $\varepsilon^{\prime}$ 和 $\tan \delta$ 曲线. (d) 1 的介电频率与峰值温度的 Arrhenius 关系曲线, 红色实线为拟合结果.

Figure 2 (a) The PXRD diagrams for 1. Inset is digital picture of the large crystal of 1. (b) The specific heat capacity test for 1. (c) Temperature-dependent dielectric constant $\left(\varepsilon^{\prime}\right.$ and $\tan \delta$ ) plots for 1 under different frequencies. (d) Arrhenius plots of the peak temperatures and frequencies for the dielectric relaxations of $\mathbf{1}$, the red line is the fitting result. 


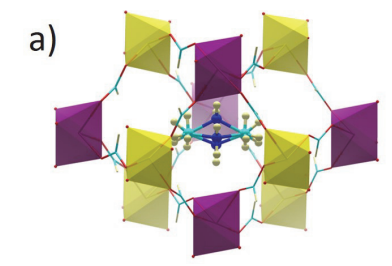

b)

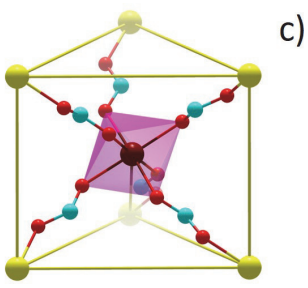

d)

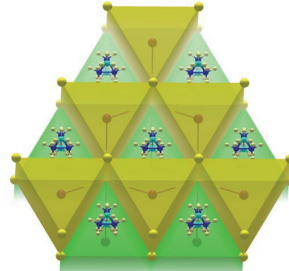

g)

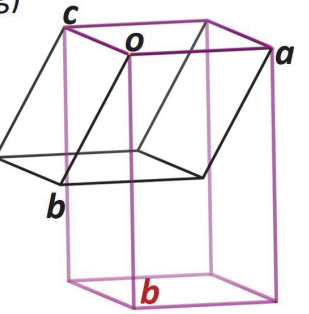

e)

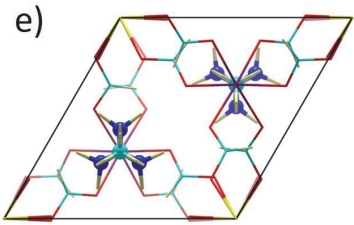

h)



i) c)

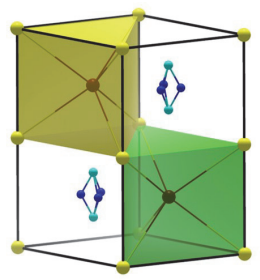

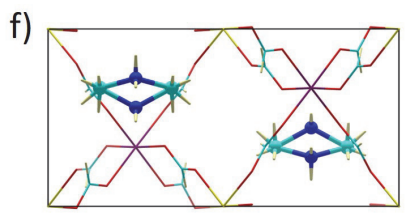



图 3 (a) 无序有机胺填充在多面体空隙的示意图. (b) 二价铁为中心和相邻三价铁构筑的超三棱柱结构. (c) 相邻超三棱柱通过共边相连的结构 示意图. (d) 超三棱柱结构和甲胺在 $c$ 方向的堆积图. (e)和(f)高温相 $c$ 和 $a$ 方向看甲胺的无序状态. (g) 相变前后晶胞变化示意图(高温相为黑色, 低 温相为粉色). (h)和(i)低温相 $c$ 和 $a$ 方向看甲胺的有序状态. 图中 $\mathrm{Fe}^{\mathrm{II}} 、 \mathrm{Fe}^{\mathrm{III}} 、 \mathrm{C} 、 \mathrm{O} 、 \mathrm{~N}$ 和 $\mathrm{H}$ 分别用紫色、黄色、青色、红色、蓝色和淡黄色表示. Figure 3 (a) The views of the polyhedral cavity with the disordered amines. (b) The trigonal prism structure constructed by the $\mathrm{Fe}^{\mathrm{II}}$ and $\mathrm{Fe}^{\mathrm{III}}$ ions. (c) The linkage of the trigonal prisms. (d) The packing modes of the trigonal prism and MA along $c$-direction. (e) and (f) The disorder MA at HT phases along $a$ and $c$-direction. (g) The primitive and conventional unit cell of HT (black) and LT (pink) phases. (h) and (i) The order DMA at LT phases along $a$ - and $c$-direction. Color scheme: $\mathrm{Fe}^{\mathrm{II}}$ (violet), $\mathrm{Fe}^{\mathrm{III}}$ (yellow), $\mathrm{C}$ (turquoises), $\mathrm{O}$ (red), $\mathrm{N}$ (blue), $\mathrm{H}$ (light yellow).

向的同一条直线上平行排列，而在 $b$ 方向相邻直线上反 平行排列, 这满足了反铁电体的要求, 1 的低温相是否 是反铁电体需要进一步的研究确认. 对比 $\mathrm{DMAFe}{ }^{\mathrm{III}} \mathrm{Fe}^{\mathrm{II}}$ 和 $\mathrm{DEAFe} \mathrm{III}^{\mathrm{III}}$ 的结构类型可知, 有机胺阳离子的形状 和尺寸影响相变过程中整体对称性破缺.

\section{3 磁性研究}

为研究化合物磁性, 我们首先测试其变温磁化率, 结果表明, 在 $0.1 \mathrm{~T}$ 的外场下, 随温度降低, 其 $\chi_{\mathrm{M}}$ 值缓 慢上升，直到 $40 \mathrm{~K}$ 左右开始急剧增加，并在 $35 \mathrm{~K}$ 达到 峰值 $1.42 \mathrm{~cm}^{3} \cdot \mathrm{mol}^{-1}$; 随温度降低, $\chi_{\mathrm{M}}$ 值急剧下降, 在 21 $\mathrm{K}(T$ 补, 定义为磁矩由正到负的转变温度)由正转负, 并 在 $3.6 \mathrm{~K}$ 达到最小值 $-1.92 \mathrm{~cm}^{3} \cdot \mathrm{mol}^{-1}$; 而在 $3.6 \sim 2 \mathrm{~K}$ 之 间 $\chi_{\mathrm{M}}$ 值略微抬升, 并在 $2 \mathrm{~K}$ 时达到 $-1.90 \mathrm{~cm}^{3} \cdot \mathrm{mol}^{-1}$ (图 $4 a)$. 同样 $\chi_{\mathrm{M}} T$ 随温度降低而降低, 从室温的 6.82 $\mathrm{cm}^{3} \cdot \mathrm{K}^{-} \cdot \mathrm{mol}^{-1}$ 降低到 $48 \mathrm{~K}$ 的一个极小值 $4.09 \mathrm{~cm}^{3} \cdot \mathrm{K} \cdot$ $\mathrm{mol}^{-1}$, 然后随温度进一步降低开始急剧上升, 并在 37 $\mathrm{K}$ 达到峰值. 在进一步冷却过程中, $\chi_{\mathrm{M}} T$ 在 $24 \mathrm{~K}$ 由正转 负, 并在 $11 \mathrm{~K}$ 时达到极小值, 然后随温度降低, $\chi_{\mathrm{M}} T$ 曲 线虽有所上升, 但在 $2 \mathrm{~K}$ 依然保持负值. $\chi_{\mathrm{M}} T$ 在低温区 $2 \sim 11 \mathrm{~K}$ 的上升主要归因于磁饱和. $\chi_{\mathrm{M}}{ }^{-1}$ 在 $50 \sim 300 \mathrm{~K}$ 的 温度区间符合居里-外斯定律, 对其进行拟合得到居里 常数 $C=9.09 \mathrm{~cm}^{3} \cdot \mathrm{K}^{\circ} \cdot \mathrm{mol}^{-1}$, 外斯常数 $\theta=-54.54 \mathrm{~K}$ (图 S1a). $C$ 值大于一个 $S=5 / 2$ 的 $\mathrm{Fe}^{\mathrm{III}}$ 和一个 $S=2$ 的纯自旋
$\mathrm{Fe}^{\mathrm{II}}$ 的理论值 $7.375 \mathrm{~cm}^{3} \cdot \mathrm{K} \cdot \mathrm{mol}^{-1}$. 这表明化合物磁矩中 存在明显的轨道角动量的贡献, 外斯常数表明化合物中 存在较强反铁磁作用. 1 的外斯常数和 $\mathrm{DMAF} \mathrm{e}^{\mathrm{III}} \mathrm{Fe}^{\mathrm{II}}$ $(-54.75 \mathrm{~K})$ 的接近, 而小于 $\mathrm{DEAFe}^{\mathrm{III}} \mathrm{Fe}^{\mathrm{II}}(-48.43 \mathrm{~K}) .1$

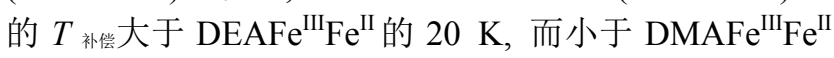
的 $29 \mathrm{~K}$. 场冷-零场冷 $(\mathrm{FC}-\mathrm{ZFC})$ 曲线表明, 1 的 $T_{\mathrm{N}}=40 \mathrm{~K}$, 证实 $C_{p}$ 在 $40 \mathrm{~K}$ 的峰是磁相变引起的(图 $\mathrm{S} 1 \mathrm{~b}$ ). 进一步采 用较大冷却场进行场冷测试, 发现在 $1 \mathrm{~T}$ 外场下, $\chi_{\mathrm{M}}$ 的 趋势和 $0.1 \mathrm{~T}$ 外场下类似, 在 $1 \mathrm{~T}$ 外场下磁化率随温度降 低先升高后转变为负值, 其 $T$ 补降低到 $17.8 \mathrm{~K}$ (图 4b). 而在 $-1 \mathrm{~T}$ 场下的 $\chi_{\mathrm{M}}$ 与 $1 \mathrm{~T}$ 下的曲线沿纵轴等于零的位 置严格对称, 其数值在 $17.8 \mathrm{~K}$ 处由负转正. 相比于 DEAFe ${ }^{\mathrm{III}} \mathrm{Fe}^{\mathrm{II}[12 \mathrm{a}]}$. $\mathrm{EAFe}^{\mathrm{III}} \mathrm{Fe}^{\mathrm{II}}\left(\mathrm{EA}=\mathrm{CH}_{3} \mathrm{CH}_{2} \mathrm{NH}_{3}{ }^{+}\right)$(Figure $\mathrm{S} 2$ )和(DMA) $\mathrm{Fe}^{\mathrm{III}} \mathrm{Fe}^{\mathrm{II}[10]}$ 分别在 $1 \mathrm{~T} 、 1 \mathrm{~T}$ 和 $0.2 \mathrm{~T}$ 的外场 下的磁化率已变为正值, 1 具有较大各向异性. 比较 $\operatorname{DEAFe}^{\mathrm{III}} \mathrm{Fe}^{\mathrm{II}}(38 \mathrm{~K}) 、 \mathrm{DMAFe}^{\mathrm{III}} \mathrm{Fe}^{\mathrm{II}}(37 \mathrm{~K})$ 和 1 的 $T_{\mathrm{N}}$ 和 0.1

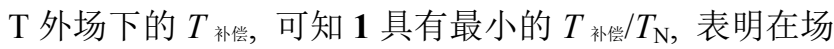
冷过程中 1 中 $\mathrm{Fe}^{\mathrm{II}}$ 有序速度最快, 而 $\mathrm{Fe}^{\mathrm{III}}$ 有序速度则最 慢. 1 是首例在 $1 \mathrm{~T}$ 冷却场下保持负磁化行为并具有明确

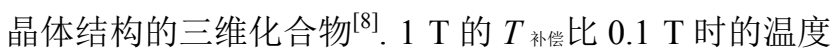
要低, 表明在冷却过程中, 较大的外加磁场进一步强化 了 $\mathrm{Fe}^{\mathrm{II}}$ 亚晶格的有序程度, 弱化了 $\mathrm{Fe}^{\mathrm{III}}$ 的有序程度. 这 种伴有磁化率由正转负的行为是典型的 $N$-型亚铁磁体 
特质, 在冷却过程中 $\mathrm{Fe}^{\mathrm{II}}$ 亚晶格磁矩首先有序并平行于 外磁场排列, 在反铁磁作用下 $\mathrm{Fe}^{\mathrm{III}}$ 的自旋磁矩反平行于 外磁场排列. 随温度进一步降低, $\mathrm{Fe}^{\mathrm{III}}$ 的磁矩逐渐有序 并在某一温度时和 $\mathrm{Fe}^{\mathrm{II}}$ 磁矩相等, 这个温度被定义为


矩和外磁场反平行排列, 由于磁各向异性的存在阻碍净 磁矩转向, 使负磁化继续保持. 而不同磁场下磁化率的 正负体现了磁场下塞曼能和各向异性的竞争. 如果各向 异性较小, 那么较低外磁场就可翻转磁矩使净磁矩平行 于磁场. 反之, 各向异性强则需更大磁场来翻转净磁矩 的方向. 同时, 根据不同场下 $2 \mathrm{~K}$ 时的磁矩，估算当 $\mathrm{A}$ 为 MA、DMA、EA 和 DEA 时, $\left[\mathrm{A}^{\mathrm{I}}\right]\left[\mathrm{Fe}^{\mathrm{II}} \mathrm{Fe}^{\mathrm{III}}\left(\mathrm{HCO}_{2}\right)_{6}\right]$ 的 场冷磁化率由正到负的转变场分别在 $1.3 \mathrm{~T} 、 0.13 \mathrm{~T} 、 0.5$ $\mathrm{T}$ 和 $0.4 \mathrm{~T}$. 负磁化的出现与耦合、各向异性、温度依赖 的亚晶格有序以及外场密切相关. 尽管 $\left[\mathrm{A}^{\mathrm{I}}\right]\left[\mathrm{Fe}^{\mathrm{II}} \mathrm{Fe}^{\mathrm{III}}-\right.$ $\left.\left(\mathrm{HCO}_{2}\right)_{6}\right]$ 中铁离子均处于八面体场配位环境, 但通过仔
细计算 ${ }^{[18]}$ 发现 $\mathrm{Fe}^{\mathrm{III}}$ 在相变前后均处于较规则的八面体 配位环境, 且具有较小的扭曲值; $\mathrm{Fe}^{\mathrm{II}}$ 的扭曲值比 $\mathrm{Fe}^{\mathrm{III}}$ 大 并随着有机胺的不同而改变. 为理解结构与磁性的关 系, 将具有明确结构的 $\left[\mathrm{A}^{\mathrm{I}}\right]\left[\mathrm{Fe}^{\mathrm{II}} \mathrm{Fe}^{\mathrm{III}}\left(\mathrm{HCO}_{2}\right)_{6}\right]$ 的部分磁性 和配位几何参数进行比较发现(表 1): 化合物的外斯常 数 $\theta$ 体现出反铁磁耦合的强弱与甲酸连接的 $\mathrm{Fe}^{\mathrm{II}}-\mathrm{O}-\mathrm{C}$ 和 $\mathrm{Fe}^{\mathrm{III}}-\mathrm{O}-\mathrm{C}$ 二面角有关 ${ }^{[19]}$, 较小的二面角利于获得较强反 铁磁耦合. 此外, 在耦合接近时, $0.1 \mathrm{~T}$ 冷却场下负磁矩 大小以及场冷磁化率由正到负转变场的大小均与低温 时 $\mathrm{Fe}^{\mathrm{II}}$ 配位构型有关. 分析表明, $\mathrm{Fe}^{\mathrm{II}}$ 配位构型越接近正 八面体, 化合物的转变场和 $0.1 \mathrm{~T}$ 冷却场下磁化率的绝 对值越大. 以上结果表明：客体分子微调框架中金属离 子配位构型可实现化合物磁性调控; 八面体构型的 $\mathrm{Fe}^{\mathrm{II}}$ 和较小耦合利于获得较大负磁化.

这种具有负磁化行为的化合物可用作正场调控的 极化翻转开关. 为了验证其可行性, 在 100 Oe 外场下,
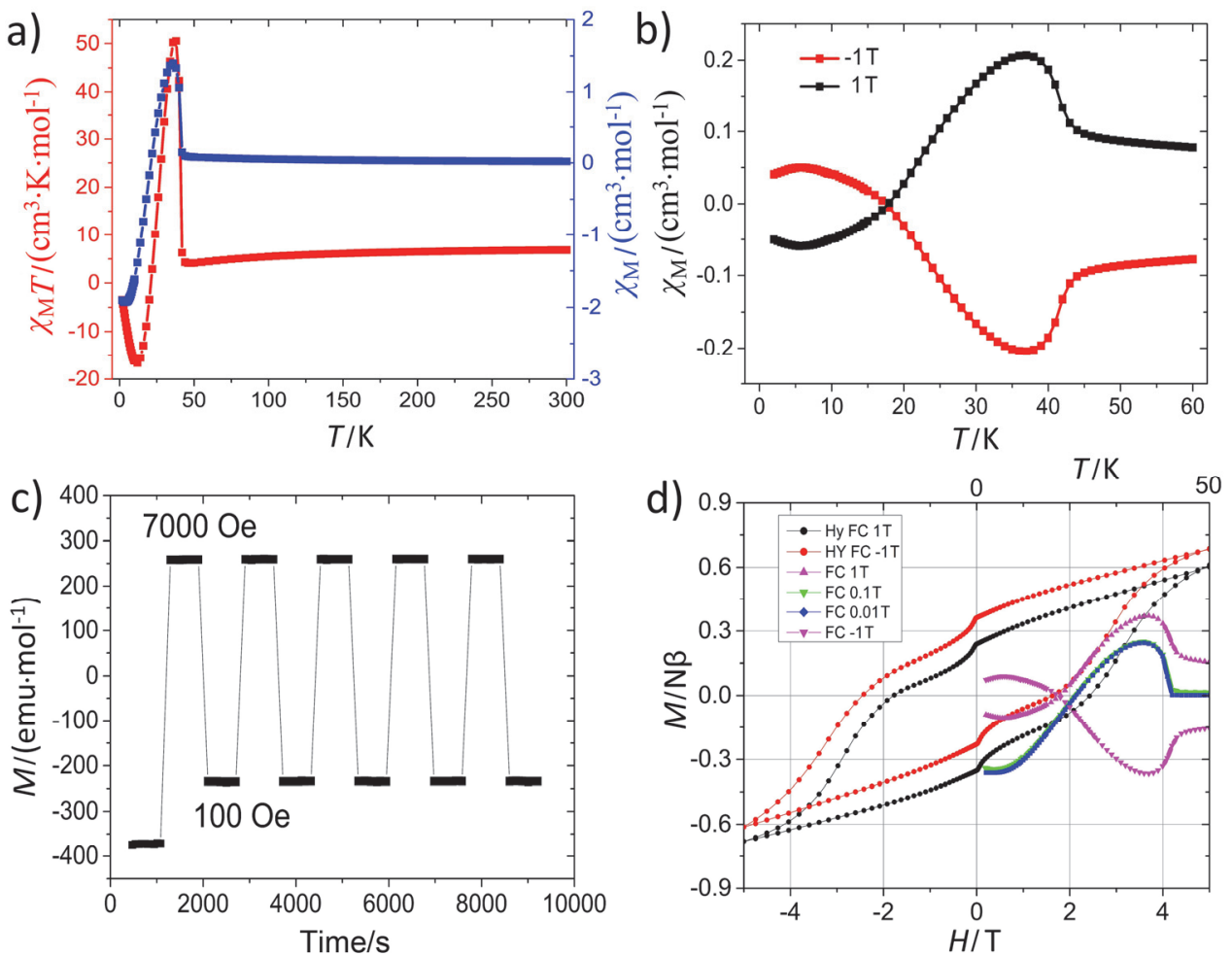

图 4 (a) 1 在 $0.1 \mathrm{~T}$ 外场下的 $\chi_{\mathrm{M}}$ vs. $T$ 和 $\chi_{\mathrm{M}} T$ vs. $T$ 曲线. (b) 1 在 $1 \mathrm{~T}$ 和 $-1 \mathrm{~T}$ 场下的场冷曲线. (c) 1 在 $100 \mathrm{Oe}$ 场冷后, $17 \mathrm{~K}$ 时 $100 \mathrm{Oe}$ 和 $7000 \mathrm{Oe}$ 下 正场调制的磁极翻转. (d) 在 $1 \mathrm{~T}$ 和 $-1 \mathrm{~T}$ 场冷后, 1 在 $2 \mathrm{~K}$ 的磁滞回线和不同外场下的场冷曲线.

Figure 4 (a) $\chi_{\mathrm{M}}$ vs. $T$ and $\chi_{\mathrm{M}} T$ vs. $T$ plots of 1 under $0.1 \mathrm{~T}$ applied field. (b) Field-cooled magnetization for 1 under $1 \mathrm{~T}$ and $-1 \mathrm{~T}$ applied fields. (c) Bipolar switching of magnetization for 1 at $17 \mathrm{~K}$ under flipped magnetic field 100 and 7000 Oe after 100 Oe magnetic field cooling. (d) $1 \mathrm{~T}$ and $-1 \mathrm{~T}$ field cooled hysteresis loops recorded at $2 \mathrm{~K}$ for $\mathbf{1}$ and field-cooled magnetization under different applied fields for $\mathbf{1}$.

表 $1 \quad\left[\mathrm{~A}^{\mathrm{I}}\right]\left[\mathrm{Fe}^{\mathrm{II}} \mathrm{Fe}^{\mathrm{III}}\left(\mathrm{HCO}_{2}\right)_{6}\right]$ 的磁性和部分配位几何参数表

Table 1 Magnetism and coordination geometry parameter of $\left[\mathrm{A}^{\mathrm{I}}\right]\left[\mathrm{Fe}^{\mathrm{II}} \mathrm{Fe}^{\mathrm{III}}\left(\mathrm{HCO}_{2}\right)_{6}\right]$

\begin{tabular}{|c|c|c|c|c|c|c|c|}
\hline $\mathrm{A}$ & $\theta / \mathrm{K}$ & $0.1 \mathrm{~T} / 1 \mathrm{~T}$ 外场 $M^{a} / \mathrm{N} \beta$ & $T_{\mathrm{N}}, T_{\text {补㒉 }} / \mathrm{K}$ & 配位夹角 ${ }^{b} /\left(^{\circ}\right)$ & $\mathrm{HT}$ 相 $\mathrm{Fe}^{\mathrm{II}}$ 扭曲 ${ }^{c}$ & 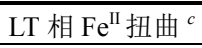 & Ref. \\
\hline MA & -54.54 & $-0.34 /-0.08$ & 40,21 & 11.02 & 0.164 & 0.182 & 本文 \\
\hline DMA & -54.75 & $-0.17 / 0.42$ & 37,29 & 12.81 & 0.194 & 0.350 & [10] \\
\hline EA & -65.00 & $-0.23 / 0.27$ & 38,25 & 9.56 & 0.146 & - & {$[12 \mathrm{~b}], \mathrm{SI}$} \\
\hline DEA & -48.43 & $-0.18 / 0.35$ & 38,20 & 13.39 & 0.173 & 0.201 & [12] \\
\hline
\end{tabular}


将 1 冷却到 $17 \mathrm{~K}$ 使其呈现负磁化状态. 保持温度不变, 增加磁场强度, 当磁场增加到 $7000 \mathrm{Oe}$ 时, 磁化率变为 正值; 将外场由 $7000 \mathrm{Oe}$ 再退回 $100 \mathrm{Oe}$, 磁化率又变为 负值(图 4c). 在 $7000 \mathrm{Oe}$ 和 $100 \mathrm{Oe}$ 下切换外磁场强度, 磁化率随外场变化, 在 $7000 \mathrm{Oe}$ 时为正, $100 \mathrm{Oe}$ 时为负, 且其数值基本保持稳定, 无明显衰减. 这表明在磁场增 加过程中, $\mathrm{Fe}^{\mathrm{II}}$ 磁矩被冻结, 并不随外场改变而改变; 当 外磁场增加到较大数值时, 克服了 $\mathrm{Fe}^{\mathrm{III}}$ 和 $\mathrm{Fe}^{\mathrm{II}}$ 间的反铁 磁耦合, 使 $\mathrm{Fe}^{\mathrm{III}}$ 的自旋方向向磁场方向偏转, 当外磁场 回到较小数值时, 反铁磁耦合又使 $\mathrm{Fe}^{\mathrm{III}}$ 自旋重回原方 向. 这种正场调制的磁极翻转有望在特殊的磁存储中获 得应用. 需要说明的是, 1 的负磁化状态在经历 7000 Oe 强外场后依然能重新恢复, 表明 $\mathbf{1}$ 中 $\mathrm{Fe}^{\mathrm{II}}$ 磁矩被冷却场 牢牢冻结, 具有较强各向异性. 同时对比不同外场下的 场冷曲线可看到, $0.01 \mathrm{~T} 、 0.1 \mathrm{~T}$ 下的场冷曲线几乎重合, 而且很快达到饱和值, 这正是亚铁磁体的特征. 更加有 趣的是, 不同于一般磁体的场冷磁矩绝对数值随外加磁 场增加而增加, 1 在 $1 \mathrm{~T}$ 场冷下磁矩的绝对值在低温时明 显低于低场下的数值, 进一步体现其 $N$-型亚铁磁体的 特征. $2 \mathrm{~K}$ 下的场冷磁滞回线具有较大的矫顽力且呈现 明显的不对称性, 向冷却过程中外加磁场方向偏转, 表 明存在正交换偏置(图 4d). 在 $1 \mathrm{~T}$ 和 $-1 \mathrm{~T}$ 冷却场下, 磁 滞回线具有相同形状, 但偏转方向不同, 以 $1 \mathrm{~T}$ 冷却场 下磁滞回线进行计算, 其有效矫顽力 $\left(H_{\mathrm{C}}{ }^{\mathrm{eff}}=\left(H^{+}-\right.\right.$ $\left.\left.H^{-}\right) / 2\right)$ 和交换偏置场 $\left(H_{\mathrm{EB}}=\left(H^{+}+H^{-}\right) / 2\right)$ 分别为 21076 $\mathrm{Oe}$ 和 $3252 \mathrm{Oe}$, 其中 $H^{+}=24328 \mathrm{Oe}, H^{-}=-17824 \mathrm{Oe}$; 同时正向和反向退磁过程中的剩磁分别为 $0.24 \mathrm{~N} \beta$ 和 $-0.35 \mathrm{~N} \beta .1$ 的交换偏置行为表明, 在场冷过程中, 自 旋冻结产生一个不随外场改变的高度各向异性内场 $\mu_{\mathrm{i}}$, 其来源可能与结构缺陷相关 ${ }^{[10]}$. 一般来说, 交换偏置行 为由铁磁层和反铁磁层界面间的耦合产生. 在冷却过程 中, 铁磁层先于反铁磁层有序, 诱导了界面处反铁磁层 中磁矩的排列 ${ }^{[20]}$. 冷却后, 反铁磁层的磁矩方向不随外 场改变而改变, 结合界面的耦合造成了交换各向异性产 生交换偏置. 交换偏置往往出现在 $N$-型亚铁磁体中, 这 可能是由于亚晶格磁有序的温度差异满足了先有序磁 矩对缺陷部分磁矩的定向排列要求. 排列后的缺陷部分 磁矩如果不随外场改变就易于形成交换偏置, 其机理需 要进一步研究.

\section{3 结论}

本工作报道一种以最小有机胺客体为抗衡离子的 砷化镍型混价态甲酸铁 $\left[\mathrm{CH}_{3} \mathrm{NH}_{3}\right]_{n}\left[\mathrm{Fe}^{\mathrm{III}} \mathrm{Fe}^{\mathrm{II}}\left(\mathrm{HCO}_{2}\right)_{6}\right]_{n}$. 1 中有机胺 $\mathrm{CH}_{3} \mathrm{NH}_{3}{ }^{+}$的有序-无序转变触发结构相变, 伴 随此结构相变, 1 展现出介电弛豫行为. 磁性研究表明, 1 是一例 $N$-型亚铁磁体, 其磁相变温度为 $40 \mathrm{~K}$; 更加有 趣的是, 1 存在较强各向异性, 使其在 $1 \mathrm{~T}$ 冷却场下依然 保持负磁化行为, 这在具有明确晶体结构的分子基磁体
中尚属首次发现. 同时 1 在低温区呈现正场调控磁极翻 转行为, 其负磁化可逆翻转磁场高达 7000 Oe. 场冷磁 滞回线研究表明该化合物具有较大矫顽力和交换偏置 场. 总之, 本研究表明 1 中存在丰富的物理性质, 其磁 性行为尤其特殊, 拓宽了负磁化和正场调控磁极翻转的 磁场范围. 1 中存在的结构相变、介电弛豫、磁极翻转、 交换偏置等功能间的耦合, 值得进一步研究. 本研究进 一步表明客体分子改换是调控结构相变、磁、电等性能 的有效手段.

\section{致谢}

感谢北京理工大学陶军教授和大连理工大学刘涛 教授在磁性研究方面的帮助.

\section{References}

[1] Kahn, O. Nature 1999, 399, 21.

[2] Kumar, A.; Yusuf, S. M. Phys. Rep. 2015, 556, 1.

[3] Néel, L. Ann. Phys. 1984, 3, 137.

[4] Markovich, V.; Fita, I.; Puzniak, R.; Martin, C.; Kikoin, K.; Wisniewski, A.; Hebert, S.; Maignan, A.; Gorodetsky, G. Phys. Rev. B 2006, 74, 174408.

[5] Ravi, P. S.; Tomy, C. V. J. Phys.: Condens. Matter. 2008, 20, 235209.

[6] Ohkoshi, S.; Hashimoto, K. J. Am. Chem. Soc. 1999, 121, 10591.

[7] Ohkoshi, S.; Abe, Y.; Fujishima, A.; Hashimoto, K. Phys. Rev. Lett. 1999, 82,1285 .

[8] (a) Nuttall, C. J.; Day, P. Chem. Mater. 1998, 10, 3050. (b) Day, P. Chem. Mater. 2003, 15, 2288. (c) Bhattacharjee, A. Chem. Mater. 2003, 15, 2287. (d) Tang, G.; He, Y.; Liang, F.; Li, S.; Huang, Y. Physica B 2007, 392, 337.

[9] Fishman, R. S.; Reboredo, F. A. Phys. Rev. Lett. 2007, 99, 217203.

[10] Zhao, J.-P.; Hu, B.-W.; Lloret, F.; Tao, J.; Yang, Q.; Zhang, X.-F.; $\mathrm{Bu}, \mathrm{X} . \mathrm{-H}$. Inorg. Chem. 2010, 49, 10390.

[11] Cañadillas-Delgado, L.; Fabelo, O.; Rodríguez-Velamazán, J. A.; Lemée-Cailleau, M.-H.; Mason, S. A.; Pardo, E.; Lloret, F.; Zhao, J.-P.; Bu, X.-H.; Simonet, V.; Colin, C. V.; Rodríguez-Carvajal, J. J. Am. Chem. Soc. 2012, 134, 19772.

[12] (a) Zhao, J. P.; Xu, J.; Han, S. D.; Wang, Q. L.; Bu, X. H. Adv. Mater. 2017, 29, 1606966. (b) Mączka, M.; Ciupa, A.; Gagor, A.; Sieradzki, A.; Pikul, A.; Ptaka, M. J. Mater. Chem. C 2016, 4, 1186.

[13] Zhao, J.-P.; Han, S.-D.; Jiang, X.; Liu, S.-J.; Zhao, R.; Chang, Z.; Bu, X.-H. Chem. Commun. 2015, 51, 8288.

[14] Zhao, J. P.; Han, S. D.; Liu, F. C. Inorg. Chem. 2019, 58, 1184

[15] (a) Yu, Y.; Shang, R.; Chen, S.; Wang, B.-W.; Wang, Z.-M.; Gao, S. Chem. Eur. J. 2017, 23, 9857. (b) Shang, R.; Chen, S.; Hu, K.-L.; Wang, B.-W.; Wang, Z.-M.; Gao, S. Chem. Eur. J. 2016, 22, 6199. (c) Shang, R.; Xu, G.-C.; Wang, Z.-M.; Gao, S. Chem. Eur. J. 2014, $20,1146$.

[16] (a) Zhao, Y.-H.; Liu, S.; Wang, B.-W.; Wang, Z.-M.; Gao, S. Chem. Eur. J. 2019, 25, 9303. (b) Sieradzkia, A.; Pawlus, S.; Tripathy, S. N.; Gągorc, A.; Ciupac, A.; Mączkac, M.; Paluch, M. Phys. Chem. Chem. Phys. 2016, 18, 8462.

[17] (a) Brown, I. D.; Altermatt, D. Acta Crystallogr. 1985, B41, 244. (b) Brese, N. E.; O'Keeffe, M. Acta Crystallogr. 1991, B47, 192.

[18] Llunell, M.; Casanova, D.; Cirera, J.; Alemany, P.; Alvarez, S. SHAPE, Version 2.1, Universitat de Barcelona, Barcelona, Spain, 2013

[19] Zhao, J.-H.; Han, S.-D.; Jiang, X.; Liu, S.-J.; Zhao, R.; Chang, Z.; Bu, X.-H. Chem. Commun. 2015, 51, 8288.

[20] (a) Sun, Y.; Cong, J.-Z.; Chai, Y.-S.; Yan, L.-Q.; Zhao, Y.-L.; Wang, S.-G.; Ning, W.; Zhang, Y.-H. Appl. Phys. Lett. 2013, 102, 172406. (b) Bora, T.; Ravi, S. J. Magn. Magn. Mater. 2014, 358, 208. (c) Li, C.; Yan, T.; Chakrabarti, C.; Zhang, R.; Chen, X.; Fu, Q.; Yuan, S.; Barasa, G. O. J. Appl. Phys. 2018, 123, 093902.

(Cheng, B.) 